Tecno Lógicas

ISSN 0123-7799

Vol. 20, No. 38, pp. 83-94

Enero -junio de 2017

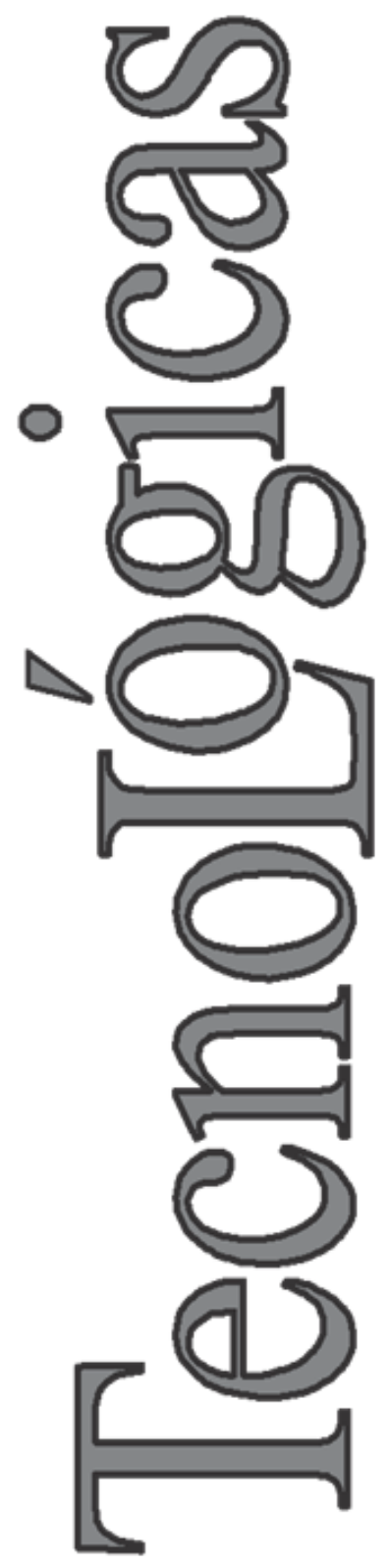

(C) Copyright 2015 por autores y Tecno Lógicas Este trabajo está licenciado bajo una Licencia Internacional Creative Commons Atribución (CC BY)

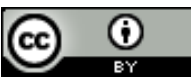

\section{Obtención de lípidos y carbohidratos a partir de microalgas mediante el diseño de medios de cultivo selectivos}

\author{
Obtaining lipids and carbohydrates from \\ microalgae via design of selective culture \\ media
}

\author{
Ana M. Ardila-Álvarez ${ }^{1}$, Yaritza López-Matos², \\ Marlys E. Vásquez-Cáceres ${ }^{3}$, Ángel D. González- \\ Delgado $^{4}$ y Andrés F. Barajas-Solano ${ }^{5}$
}

Recibido: 07 de julio de 2016, Aceptado: 28 de noviembre de 2016

Cómo citar / How to cite

Ana M. Ardila-Álvarez, Yaritza López-Matos, Marlys E. VásquezCáceres, Ángel D. González-Delgado y Andrés F. Barajas-Solano, "Obtención de lípidos y carbohidratos a partir de microalgas mediante el diseño de medios de cultivo selectivos", Tecno Lógicas, vol. 20, no. 38, pp. 83-94 enero-junio, 2017.

1 MSc. en Ingeniería Química, Escuela de Ciencias Agrícolas Pecuarias y del Medio Ambiente -ECAPMA, Universidad Nacional Abierta y a Distancia, Bucaramanga-Colombia, an.ardila@unad.edu.co

2 Ingeniera Química, Universidad Industrial de Santander, Bucaramanga-Colombia, sayaloma@hotmail.com

3 Ingeniera Química, Universidad Industrial de Santander, Bucaramanga-Colombia, mevc9@hotmail.com

4 PhD. en Ingeniería Química, Facultad de Ingeniería, Universidad de Cartagena, Cartagena-Colombia, agonzalezd1@unicartagena.edu.co

$5 \mathrm{PhD}$. en Ingeniería Química, Grupo Ambiental de Investigación Aplicada-GAIA, Facultad de Ingenierías, Universidad de Santander UDES, Bucaramanga-Colombia, an.barajas@mail.udes.edu.co 


\section{Resumen}

La producción sostenible de biorrefinerías a partir de microalgas presenta varias complicaciones técnicas a diferentes niveles, entre los que se encuentra la maximización de la productividad de bloques energéticos, como carbohidratos y lípidos, que sean materia prima para biodiesel y bioetanol. Una alternativa para aumentar la productividad de bloques energéticos es el uso de cultivos alternativos a los medios químicos tradicionales, los cuales se han basado en fuentes de carbono, fosforo, nitrógeno y microelementos. Este trabajo presenta el diseño de dos medios de cultivo mixotrófico con diferentes fuentes de carbono, nitrógeno y fósforo, a fin de evaluar la producción de carbohidratos y lípidos a partir de Chlorella vulgaris. Los medios de cultivo se diseñaron a diferentes concentraciones de nitrato de sodio, fosfato de potasio y acetato de sodio/carbonato de amonio como fuente de carbono. Además, se realizaron diagramas de Pareto y Superficies de Respuesta utilizando el software estadístico STATISTICA 7.0, a fin de conocer la influencia significativa de las variables de estudio sobre la producción de metabolitos. Los resultados mostraron que la concentración de los nutrientes en los cultivos mixotroficos afecta la producción de metabolitos, para el caso de la obtención de carbohidratos, el acetato, el carbonato y el fosfato ejercieron un efecto positivo en su producción. Para la producción de lípidos, cuando el medio de cultivo contenía acetato, no se presentó variable alguna que influyera significativamente, mientras que, para el cultivo con carbonato de amonio, el nitrato y las interacciones carbonato-fosfato, nitrato-fosfato ejercieron una influencia significativa en la producción de este metabolito.

\section{Palabras clave}

Cultivos mixotróficos, chlorella vulgaris, nutrientes, carbohidratos, lípidos.

\section{Abstract}

Sustainable production of microalgae biorefineries presents several technical bottlenecks in different levels, including maximization of productivity of energy blocks as carbohydrates and lipids, which can be used as feedstocks for biodiesel and bioethanol production. An alternative for increasing productivity of energy blocks is the use of alternative crops to traditional chemical media, which are based on carbon, phosphorus, nitrogen sources and microelements. This work presents the design of two mixotrophic crops were designed at different concentrations of carbon, nitrogen and phosphate sources with the aim of evaluating the carbohydrates and lipids production from Chlorella vulgaris. The culture media were designed at different concentrations of sodium nitrate, potassium phosphate and sodium acetate / ammonium carbonate as carbon source. In addition, Pareto charts and Response Surface were performed using the statistical software STATISTICA 7.0, in order to know the significant influence of study variables on metabolites production. Results showed that the concentration of nutrients in the mixotrophic cultures affect the production of metabolites, for the case of carbohydrates production, acetate, carbonate and phosphate had a positive effect on it. Regarding lipids production, when the culture media contained acetate, there was not any variable that influenced significantly, whereas for the cultivation with ammonium carbonate, nitrate and interactions carbonate-phosphate, nitratephosphate had a significant influence on production of this metabolite.

\section{Keywords}

Mixotrophic cultures, chlorella vulgaris, nutrients, carbohydrates, lipids. 


\section{INTRODUCCIÓN}

La utilización de microalgas como materia prima para el desarrollo de biorefinerías se ha estudiado con bastante interés en los últimos años, debido al potencial de esta materia prima para la obtención de biocombustibles y bioproductos [1], sin embargo, aún se tienen diversos cuellos de botella en diferentes escalas que deben ser superados. Con respecto al cultivo, este se ha llevado a cabo de forma natural o artificial, desde lagos y estanques abiertos hasta fotobioreactores altamente complejos y controlados [2]. Sin embargo, para lograr una productividad por área de cultivo a escala comercial con una conversión eficiente de la energía, se deben diseñar sistemas de cultivos sostenibles y rentables. En los cultivos heterótrofos es necesario mantener parámetros como temperatura, $\mathrm{pH}$ y concentración de nutrientes dentro de los límites fisiológicos de la microalga a fin de favorecer su crecimiento. Algunas especies de microalgas utilizan fuentes de carbono orgánico como acetato, glucosa y glicerol [3]. Aunque este tipo de cultivos permiten el uso eficiente de la luz, a gran escala, el crecimiento es lento debido a su limitada penetración a través del espesor de la columna de agua y la alta densidad de células [4]. Una alternativa viable para algunas especies es el uso de cultivos mixotróficos, en los cuales se pueden utilizar fuentes de carbono orgánicas e inorgánicas en presencia de luz que son asimiladas simultáneamente con el $\mathrm{CO} 2$ mediante metabolismos fotosintético y respiratorio, llevando a una elevada producción de biomasa [4], [5]. Otra de las ventajas que representa este tipo de cultivo es que se asegura una tasa de productividad de lípidos y biomasa de 3 a 10 veces superior a la generada en cultivos fotoautótrofos en biorreactores [6],[3]. Sin embargo, en este tipo de cultivos es necesario controlar la concentración de los nutrientes debido a que ciertos cambios pueden aumentar o inhibir la tasa de crecimiento de las algas
[7]. Zhu et al. [8] reportaron que el contenido de lípidos puede aumentar debido al estrés generado por la limitación de nitrógeno y fósforo, o de nitrógeno solamente [9]. Esta situación se encuentra asociada a baja productividad de biomasa, por lo que es necesario contar con una fuente alterna de nitrógeno que permita mantener una alta tasa de crecimiento de los microorganismos [10]. Por otra parte, en un estudio de obtención de biodiesel en Chlorella zofingiensis, la glucosa resultó ser la mejor fuente de carbono para este propósito y para el caso de cultivo de Phaeodactylum tricornutum, se obtuvo la más alta productividad de biomasa utilizando glicerol, acetato y glucosa en el medio mixotrófico [11]. De hecho, el crecimiento mixotrófico utilizando glicerol puede exceder el observado en cultivos heterótrofos [9]. Finalmente, algunas especies como Chlorella, Chlamydomonas y Nannochloropsis han demostrado altas tasas de acumulación de lípidos bajo fuentes de carbono y nitrógeno a distintas concentraciones [12]. Por lo tanto, en este trabajo se evaluaron simultáneamente diferentes fuentes de carbono, nitrógeno y fósforo en un medio de cultivo Bold Basal, como parte de cultivos mixotróficos diseñados con el fin de mejorar la producción de carbohidratos y lípidos a partir de Chlorella vulgaris UTEX 1803.

\section{METOdOLOGía}

\subsection{Microalga}

Chlorella vulgaris UTEX 1803 fue adquirida de la Universidad de Texas, USA y cultivada en medio Bold Basal modificado [13] durante 15 días a $25 \pm 2{ }^{\circ} \mathrm{C}, \mathrm{pH} 7$, sin ningún suministro complementario de $\mathrm{CO}_{2}$ e iluminado con lámparas fluorescentes con un ciclo de luz-oscuridad 12-12 horas. 


\subsection{Medio de cultivo}

Cada litro de medio de cultivo empleado tenía la composición de macronutrientes y micronutrientes registrada en la Tabla 1.

\subsection{Bioreactor}

Se usaron biorreactores rectangulares con dimensiones $20 \mathrm{~cm}$ largo, $20 \mathrm{~cm}$ altura y $10,5 \mathrm{~cm}$ ancho, donde cada ex-tremo inferior contaba con un suministro de aire continuo por burbujeo, con el fin de proveer aire a los cultivos y garantizar que todas las células estuviesen expuestas a la luz y los nutrientes del medio.

\subsection{Diseño de cultivos mixotróficos}

A fin de mejorar la productividad de carbohidratos y lípidos, se diseñaron cultivos mixotróficos con base en dos fuentes de carbono (acetato de sodio y carbonato de amonio), nitrógeno (NaNO3) y fósforo
(K2HPO4 y K2HPO4), manteniendo la concentración de los macronutrientes y micronutrientes constantes según las especificaciones del Medio Bold Basal.

Para determinar el efecto de las variables propuestas se evaluaron 3 concentraciones de los diferentes nutrientes (Tabla 2 y 4 ) empleando diseño experimental de composición central no facto-rial 33 empleando el software STATISTICA 7.0 (StatSoft, Inc. 2004). Cada uno de los experimentos se realizó por duplicado y tuvo una duración máxima de 15 días. Como control se empleó el medio Bold Basal Modificado sin adición de carbono y con las concetraciones de nitrogeno y fosforo presente en la Tabla 1 .

La Tabla 5 presenta la composición del segundo cultivo compuesto por carbonato de amonio, nitrato de sodio y fosfato de potasio en las mismas concentraciones del primer cultivo.

\begin{tabular}{|c|c|c|}
\hline Tipo de nutriente & Nombre & Concentración final en medio (moles) \\
\hline \multirow{6}{*}{ Macronutrientes } & $\mathrm{NaNO}_{3}$ & $2,94 \times 10^{-3}$ \\
\hline & $\mathrm{MgSO}_{4} \cdot 7 \mathrm{H}_{2} \mathrm{O}$ & $3,04 \times 10^{-4}$ \\
\hline & $\mathrm{NaCl}$ & $4,28 \times 10^{-4}$ \\
\hline & $\mathrm{K}_{2} \mathrm{HPO}_{4}$ & $4,31 \times 10^{-4}$ \\
\hline & $\mathrm{KH}_{2} \mathrm{PO}_{4}$ & $1,29 \times 10^{-3}$ \\
\hline & $\mathrm{CaCl}_{2} \cdot 2 \mathrm{H}_{2} \mathrm{O}$ & $1,70 \times 10^{-4}$ \\
\hline \multirow{2}{*}{ Solución Alcalina EDTA } & EDTA & $1,71 \times 10^{-4}$ \\
\hline & $\mathrm{KOH}$ & $5,53 \times 10^{-4}$ \\
\hline \multirow[b]{2}{*}{ Solución acidificada de Hierro } & $\mathrm{FeSO}_{4} \cdot 7 \mathrm{H}_{2} \mathrm{O}$ & \\
\hline & $\mathrm{H}_{2} \mathrm{SO}_{4}$ & $1,79 \times 10^{5}$ \\
\hline \multirow[t]{3}{*}{ Solución de Boro } & $\mathrm{H}_{3} \mathrm{BO}_{3}$ & $1,85 \times 10^{-4}$ \\
\hline & $\mathrm{ZnSO}_{4} \cdot 7 \mathrm{H}_{2} \mathrm{O}$ & $3,07 \times 10^{-5}$ \\
\hline & $\mathrm{MnCl}_{2} \cdot 4 \mathrm{H}_{2} \mathrm{O}$ & $7,28 \times 10^{-6}$ \\
\hline \multirow[t]{3}{*}{ Micronutrientes } & $\mathrm{MoO}_{3}$ & $4,93 \times 10^{-6}$ \\
\hline & $\mathrm{CuSO}_{4} \cdot 5 \mathrm{H}_{2} \mathrm{O}$ & $6,29 \times 10^{-6}$ \\
\hline & $\mathrm{Co}\left(\mathrm{NO}_{3}\right)_{2} \cdot 6 \mathrm{H}_{2} \mathrm{O}$ & $1,68 \times 10^{-6}$ \\
\hline
\end{tabular}




\subsection{Cuantificación de carbohidratos}

Para esta cuantificación se utilizó el método colorimétrico fenolácido sulfúrico [14]. Se tomó $1 \mathrm{~mL}$ de medio de cultivo, al cual se le adicionaron $0,5 \mathrm{~mL}$ de fenol al $5 \%$ y $2,5 \mathrm{~mL}$ de ácido sulfúrico al $95 \%$, seguido de homogenización.

Tabla 2. Diseño experimental para el medio de cultivo con acetato de sodio, nitrato de sodio y buffer de potasio. Fuente: Autores.

\begin{tabular}{ccc}
\hline $\begin{array}{c}\text { Acetato de } \\
\text { sodio, mM }\end{array}$ & $\begin{array}{c}\text { Nitrato } \\
\text { sodio, mM }\end{array}$ & $\begin{array}{c}\text { Buffer de potasio }\left(\mathrm{K}_{2} \mathrm{HPO}_{4}\right. \\
\left.+\mathrm{KH}_{2} \mathrm{PO}_{4}\right), \mathrm{mM}\end{array}$ \\
\hline 10 & 0,98 & 0,57 \\
20 & 1.96 & 1,15 \\
30 & 2,94 & 1,72 \\
\hline
\end{tabular}

Se midió la absorbancia de la muestra a $480 \mathrm{~nm}, 485 \mathrm{~nm}$ y $490 \mathrm{~nm}$ a fin de identificar la xilosa, arabinosa, glucosa y fructosa presentes en la biomasa mediante el uso de curvas de concentración previamente preparadas. Por último, se calculó el contenido de carbohidratos de las muestras en miligramos de carbohidrato por litro [15].
Tabla 4. Diseño experimental para el medio de cultivo con acetato de sodio, nitrato de sodio y buffer de potasio.

\begin{tabular}{ccc}
\multicolumn{3}{c}{ Fuente: Autores. } \\
\hline $\begin{array}{c}\text { Acetato de } \\
\text { sodio, mM }\end{array}$ & $\begin{array}{c}\text { Nitrato } \\
\text { sodio, } \mathrm{mM}\end{array}$ & $\begin{array}{c}\text { Buffer de potasio } \\
\left(\mathrm{K}_{2} \mathrm{HPO}_{4}+\mathrm{KH}_{2} \mathrm{PO}_{4}\right), \\
\mathrm{mM}\end{array}$ \\
\hline 10 & 0,98 & 0,57 \\
20 & 1.96 & 1,15 \\
30 & 2,94 & 1,72 \\
\hline
\end{tabular}

\subsection{Cuantificación de lípidos}

La cuantificación de lípidos se realizó siguiendo el procedimiento de Chen \& Vaidyanathan (2012) [16] el cual es un método desarrollado para la cuantificación de lípidos en pequeñas cantidades de biomasa. Se tomaron 0,05 g de biomasa los días 5,10 y 15 de cultivo, se suspendieron en $100 \mu \mathrm{L}$ de Tris-HCl $1 \mathrm{M}$ a $\mathrm{pH} 8$ y se añadieron $2.400 \mu \mathrm{L}$ de reactivo de saponificación (25\% de metanol en $1 \mathrm{M} \mathrm{NaOH})$ y $250 \mathrm{mg}$ de perlas de vidrio de $0,1 \mathrm{~mm}$.

Tabla 3. Diseño experimental para el medio de cultivo con acetato de sodio, nitrato de sodio $\mathrm{y}$ fosfato de potasio. Fuente: Autores.

\begin{tabular}{ccccc}
\hline Número de tratamiento & Acetato de sodio, $\mathrm{mM}$ & Nitrato sodio, $\mathrm{mM}$ & Fosfato de potasio, $\mathrm{mM}$ & Relación C/N/P \\
\hline 1 & 10 & 2,94 & 0,57 & $1 / 0,17 / 0,07$ \\
2 & 10 & 0,98 & 1,72 & $1 / 0,05 / 0,22$ \\
3 & 30 & 2,94 & 1,72 & $1 / 0,06 / 0,07$ \\
4 & 30 & 0,98 & 0,57 & $1 / 0,02 / 0,02$ \\
5 & 20 & 1,96 & 1,15 & $1 / 0,05 / 0,07$ \\
6 & 10 & 2,94 & 1,72 & $1 / 0,17 / 0,22$ \\
7 & 10 & 0,98 & 0,57 & $1 / 0,05 / 0,07$ \\
8 & 30 & 2,94 & 0,57 & $1 / 0,06 / 0,02$ \\
9 & 30 & 0,98 & 1,72 & $1 / 0,02 / 0,07$ \\
10 & 3,27 & 1,96 & 1,15 & $1 / 0,33 / 0,45$ \\
11 & 36,73 & 1,96 & 1,15 & $1 / 0,03 / 0,04$ \\
12 & 20 & 3,6 & 1,15 & $1 / 0,1 / 0,07$ \\
13 & 20 & 0,32 & 1,15 & $1 / 0,01 / 0,07$ \\
14 & 20 & 1,96 & 0,19 & $1 / 0,05 / 0,01$ \\
15 & 20 & 1,96 & 2,11 & $1 / 0,05 / 0,13$ \\
\hline
\end{tabular}


Posteriormente, se realizó la ruptura celular mediante agitación vigorosa por 3 min y se añadieron $2.500 \mu \mathrm{l}$ del reactivo de saponificación. La mezcla se saponificó en baño maría a $90^{\circ} \mathrm{C}$ durante 30 min y se agitó con vortex cada 5 min. Las muestras se enfriaron a temperatura ambiente, para luego mezclar $3.000 \mu \mathrm{L}$ de estas con 4.500 $\mu \mathrm{L}$ de disolvente de cloroformo y metanol (2:1) en un tubo Eppendorf por 2 min. Posteriormente, se centrifugaron a $5.000 \mathrm{rpm}$ durante 2 min y se tomó un volumen de $2.500 \mu \mathrm{L}$ de la fase orgánica, el cual fue transferido a un nuevo tubo Eppendorf que contenía reactivo de cobre. Después de este paso, se transfirieron $1.500 \mu \mathrm{L}$ de la fase orgánica a una cubeta de cuarzo y se midió la absorbancia a $260 \mathrm{~nm}$ usando un espectrofotómetro

\subsection{Análisis estadístico}

A fin de conocer la influencia significativa positiva o negativa de las variables de estudio sobre los datos obtenidos en el diseño de experimentos se realizaron dia- gramas de Pareto, así como Superficies de Respuesta con base en los resultados arrojados por los diagramas, de modo que se pudiese observar el efecto que tienen las interacciones entre las variables significativas con respecto a la producción de metabolitos.

\section{RESULTADOS Y DISCUSIÓN}

\subsection{Producción de carbohidratos}

En la Fig. 1 se presenta la concentración de carbohidratos para las dos modificaciones del medio después de 15 días de cultivo.

Para el medio de cultivo con acetato de sodio, se observa que con los tratamientos T5 y T10 se obtuvieron las mayores concentraciones de carbohidratos (73 y $72 \%$ $\mathrm{p} / \mathrm{p}$ respectivamente) las menores concentraciones se obtuvieron en los tratamientos T11 y T12. A diferencia del acetato, solo uno de los experimentos (T2) obtuvo porcentajes de carbohidratos mayores al 50\%

Tabla 5. Diseño experimental para el medio de cultivo con carbonato de amonio, nitrato de sodio y fosfato de potasio. Fuente: Autores.

\begin{tabular}{ccccc}
\hline Número de tratamiento & Carbonato de Amonio, $\mathrm{mM}$ & Nitrato sodio, $\mathrm{mM}$ & Fosfato de potasio, $\mathrm{mM}$ & Relación C/N/P \\
\hline 1 & 1,07 & 2,94 & 0,57 & $1 / 0,39 / 0,16$ \\
2 & 1,07 & 0,98 & 1,72 & $1 / 0,13 / 0,52$ \\
3 & 3,22 & 2,94 & 1,72 & $1 / 0,13 / 0,17$ \\
4 & 3,22 & 0,98 & 0,57 & $1 / 0,04 / 0,05$ \\
5 & 2,15 & 1,96 & 1,15 & $1 / 0,13 / 0,17$ \\
6 & 1,07 & 2,94 & 1,72 & $1 / 0,39 / 0,52$ \\
7 & 1,07 & 0,98 & 0,57 & $1 / 0,13 / 0,16$ \\
8 & 3,22 & 2,94 & 0,57 & $1 / 0,13 / 0,05$ \\
9 & 3,22 & 0,98 & 1,72 & $1 / 0,04 / 0,17$ \\
10 & 0,35 & 1,96 & 1,15 & $1 / 0,77 / 1,04$ \\
11 & 3,95 & 1,96 & 1,15 & $1 / 0,07 / 0,09$ \\
12 & 2,15 & 3,6 & 1,15 & $1 / 0,23 / 0,17$ \\
13 & 2,15 & 0,32 & 1,15 & $1 / 0,02 / 0,17$ \\
14 & 2,15 & 1,96 & 0,19 & $1 / 0,13 / 0,03$ \\
\hline & 2,15 & 1,96 & 2,11 & $1 / 0,13 / 0,31$ \\
\hline
\end{tabular}




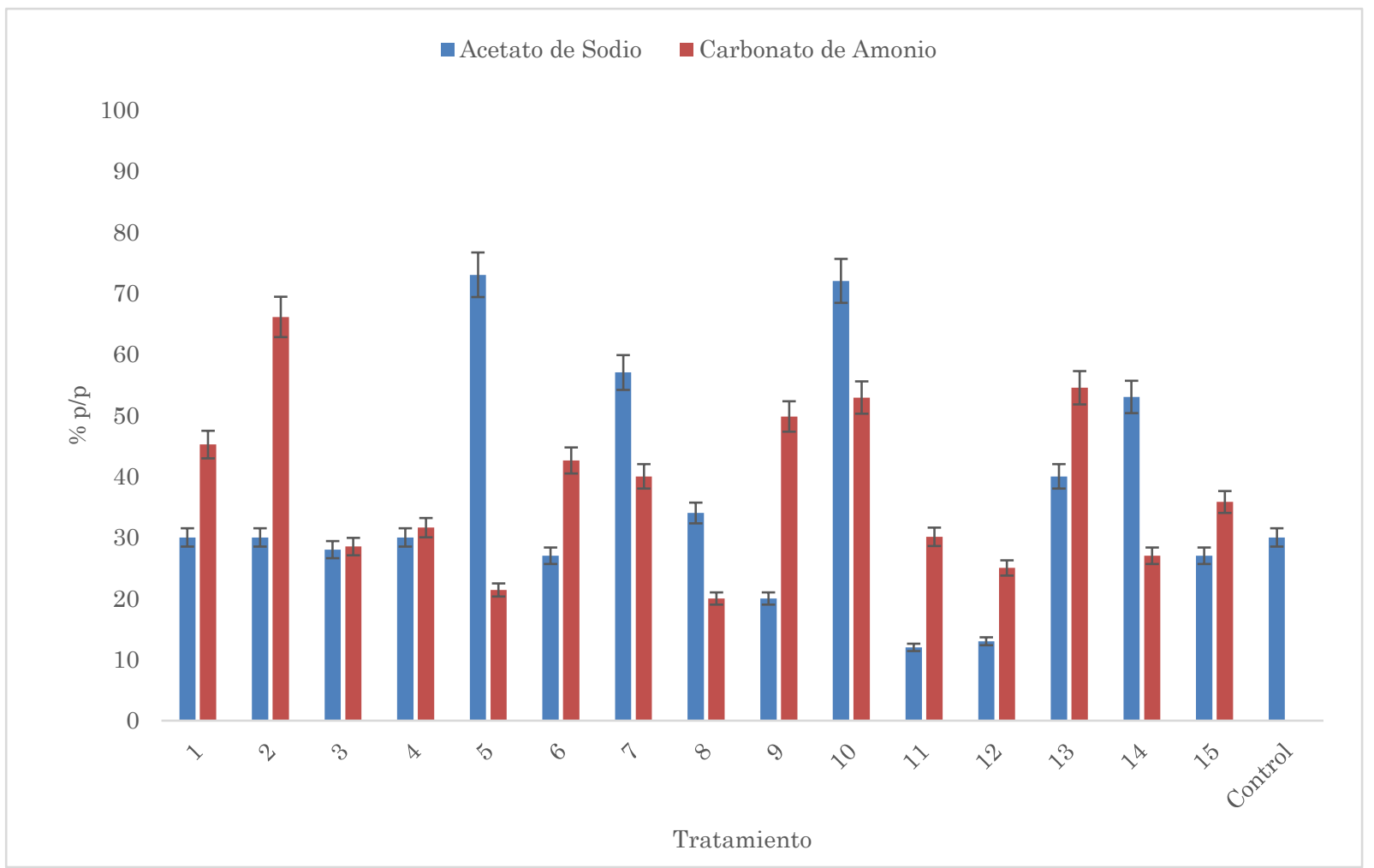

Fig. 1. Concentración de carbohidratos totales empleando acetato de sodio y carbonato de amonio después de 15 días. Fuente: Autores.

con un valor de $66 \% \mathrm{p} / \mathrm{p}$.

La producción de carbohidratos es mayor a la reportada por Dragone, Fernández, Abreu, Vicente, \& Texeira, (2011) [17] donde el rendimiento máximo fue de $41 \%$, empleando urea como fuente de nitrógeno para el medio de cultivo de C. vulgaris. Asimismo, el rendimiento resultó ser mayor al obtenido por Yanna, Nicolás, \& Yi, (2009) [18], quienes reportaron un porcentaje de carbohidratos del $44 \%$ empleando glucosa al $1 \%$ en el medio como fuente de carbono. En la Fig. 2 (a) se observa que la única variable que tiene influencia significativa $(p=0,05)$ es el acetato de sodio, el cual afecta inversamente proporcional (3.27211) la producción de carbohidratos, es decir, que a menor concentración de acetato $(<30 \mathrm{mM})$, la producción de carbohidratos se hace mayor, obteniendo concentraciones de hasta $1 \mathrm{~g} / \mathrm{L}$.

En cuanto al cultivo con carbonato de amonio, la Fig. 1 (b) muestra que únicamente la concentración del carbonato y fosfato ejercen un efecto positivo en la producción de carbohidratos en la biomasa, mientras que las interacciones nitratofosfato, carbonatonitrato y carbonatofosfato no representan una influencia positiva en la producción de este metabolito. La Fig. 2 muestra que el medio de cultivo con acetato de sodio es selectivo hacia la producción de carbohidratos. Sin embargo, es necesario suministrar concentraciones promedio de acetato $(20 \mathrm{mM})$, nitrato $(1,96$ $\mathrm{mM})$ y fosfato $(1,08 \mathrm{mM})$ para obtener altas concentraciones de este metabolito. Según Estévez et al., (2013) [4] el uso de acetato de sodio es problemático en medios de cultivo debido a que su efecto es dependiente de la concentración. En este sentido, algunos autores como Degrenne et al., (2010) [19] encontraron que las concentraciones por encima de $1 \mathrm{~g} / \mathrm{L}$ pueden causar la inhibición del crecimiento por intoxicación. Por otra parte, cuando el nitrógeno se agota en el medio de cultivo, la célula utiliza el nitrógeno restante en enzimas y estructuras 
Obtención de lípidos y carbohidratos a partir de microalgas mediante el diseño de medios de cultivo selectivos

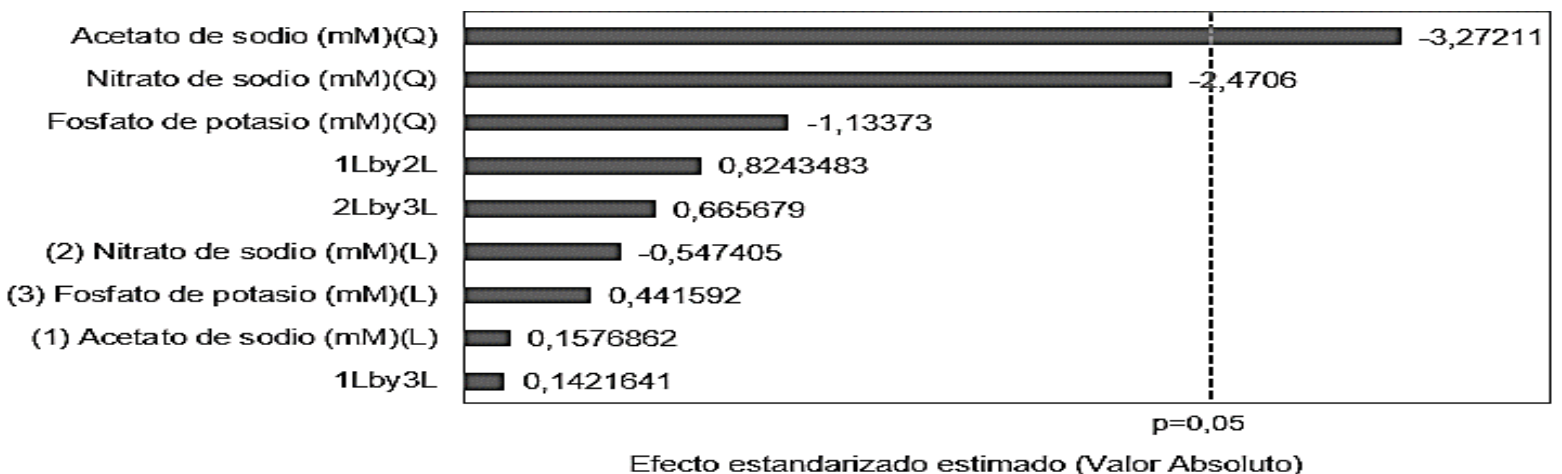

(a)

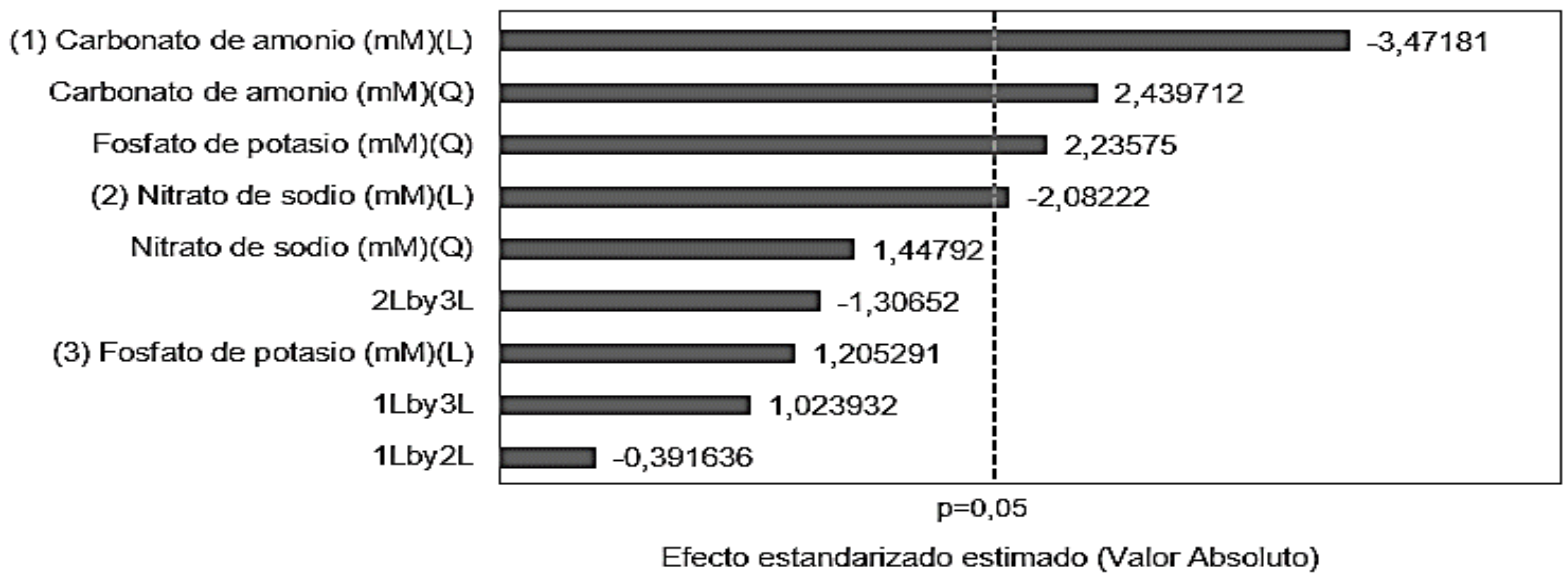

(b)

Fig. 2. Diagrama de Pareto para la producción de carbohidratos con a) acetato de sodio y b) carbonato de potasio como fuente de carbono. Fuente: Autores.

celulares esenciales, por lo que una parte del contenido de dióxido de carbono se convierte en lípidos y carbohidratos [20], en un estudio previo desarrollado por los autores se muestra que para el desarrollo de una biorefinería basada en microalgas atendiendo una composición mínima rentable bajo los parámetros establecidos el contenido de carbohidratos para la producción de la fracción bioetanol debe ser tan alta como sea posible y complementada con la obtención de algún producto de valor agregado como ácidos grasos poliinsaturados en una relación mínima de 1 (PUFAs):27,4 (bioetanol) [21].]

\subsection{Producción de lípidos}

En la Tabla 5 se observa que con el tratamiento T13 para el cultivo con acetato de sodio, se alcanzó la máxima concentración de lípidos $(0,0081 \mathrm{~g} / \mathrm{L})$ correspondiente al $0,34 \%$ de la biomasa. Por otra parte, se observa que los tratamientos T1 y T13 con carbonato de amonio permitieron obtener los mayores contenidos de concentración de lípidos $(0,0041 \mathrm{~g} / \mathrm{L}$ y $0,0031 \mathrm{~g} / \mathrm{L}$, respectivamente). El bajo porcentaje obtenido para ambos casos $(0,28$ y $0,3 \%)$ el cual no es superior al obtenido en el cultivo sin modificar $(10 \% \mathrm{p} / \mathrm{p}$ para el control) es atribuido a las condiciones del medio de cultivo (dos fuentes de nitrógeno, carbonato de amonio y nitrato de sodio) y a la forma en que la microalga se adapta a las condiciones de cultivo. El porcentaje de lípidos obtenido en este trabajo es bajo comparado con el obtenido por Porras \& Prada, (2012) [22] (21\%) en un medio de cultivo Bold Basal modificado con nitrato de sodio y acetato de sodio. 


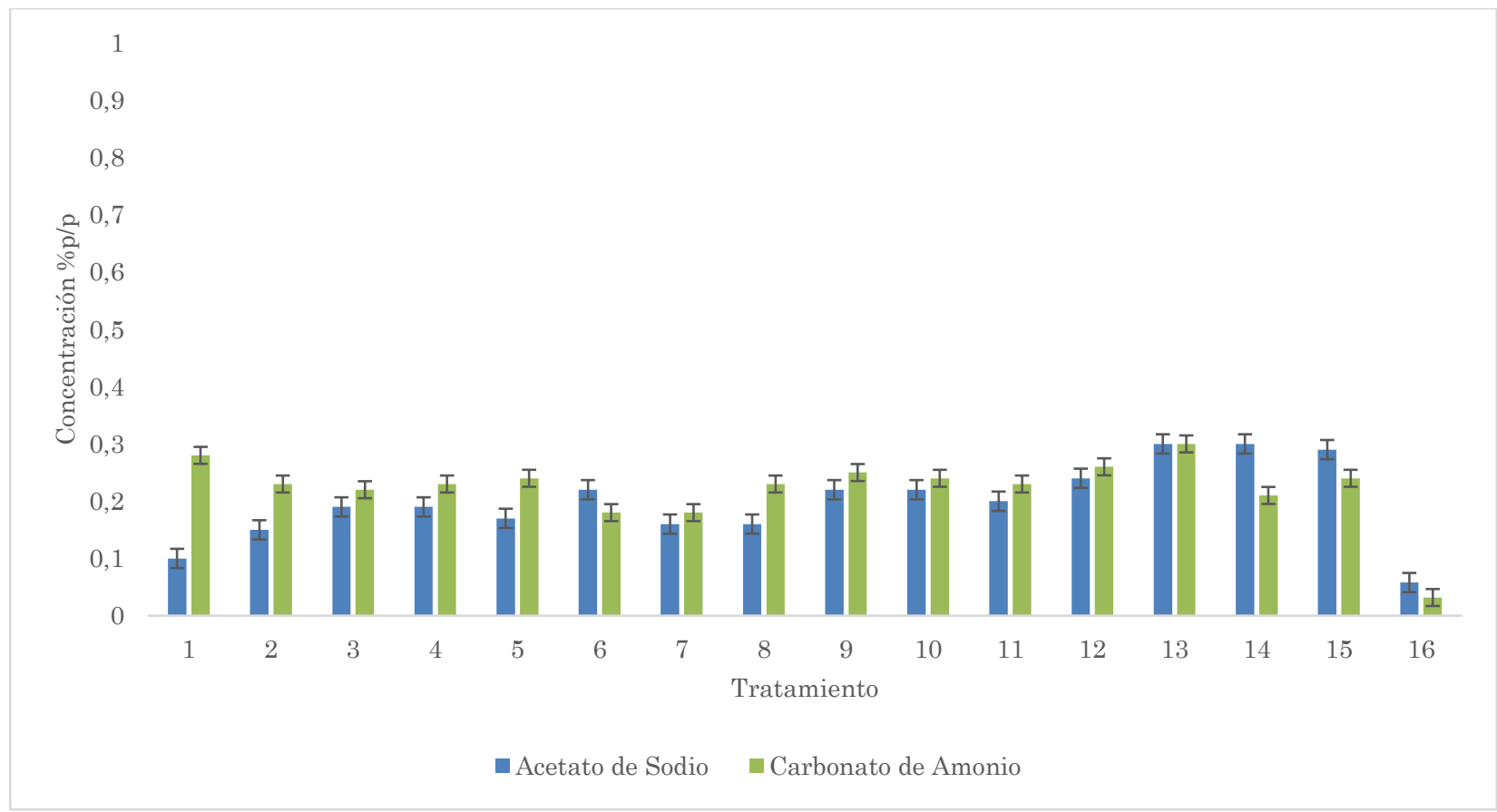

Fig. 3. Concentración de lípidos totales empleando acetato de sodio y carbonato de amonio después de 15 días. Fuente: Autores.

Los resultados obtenidos en el presente estudio revelaron que la variación simultánea de la fuente de carbono (acetato o carbonato), nitrato y fosfato provocan una disminución general de este metabolito, lo cual está de acuerdo con lo presentado por Estévez et al., (2013) [4], quienes demostraron que la regulación apropiada de la cantidad de carbono y nitrógeno no solo puede aumentar la producción de biomasa, sino también promover la deposición de ciertos metabolitos como lípidos. La alta concentración de lípidos potencia el desarrollo de biorefinerias basadas en microalgas por diferentes vías, ya sea por la producción in vivo de lípidos y alcanos vía secreción, o la extracción y transesterificación de estos para la producción de biodiesel, o por la vía térmica para la producción de precursores de biocombustibles como biocrudo, en conjunto con un alto porcentaje de carbohidratos, la selección de la ruta más adecuada de procesamiento de microalgas con alto porcentaje de carbohidratos y lípidos bajo el concepto de biorefinería puede realizarse mediante un enfoque de superestructura [23], dado el alto número de alternativas de procesamiento, en esta investigación el porcentaje de lípidos obtenido es significativamente bajo en comparación a los otros metabolitos, por lo cual las rutas de aprovechamiento integral deben enfocarse en la vía carbohidratos.

\section{CONCLUSIONES}

Los resultados obtenidos permiten concluir que la concentración de los nutrientes en los cultivos mixotróficos afecta la producción de metabolitos. El acetato, el carbonato $\mathrm{y}$ el fosfato ejercieron una influencia significativa en la producción de carbohidratos, donde la primera variable presentó un efecto inverso y las dos últimas uno directamente proporcional. En la producción de lípidos, no existe variable que influya significativamente en el cultivo con acetato de sodio, mientras que, para el cultivo con carbonato de amonio, el nitrato y las interacciones carbonato-fosfato, nitrato-fosfato ejercieron una influencia significativa en la producción de este metabolito. 


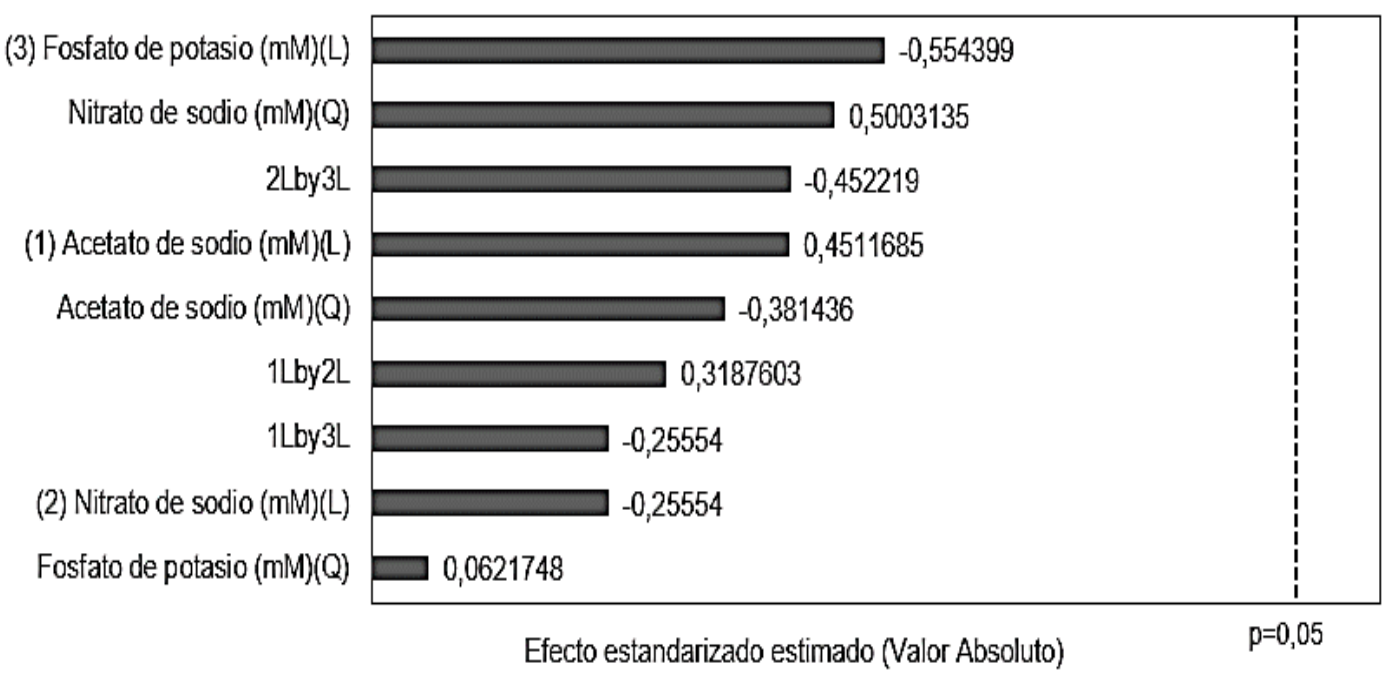

(a)

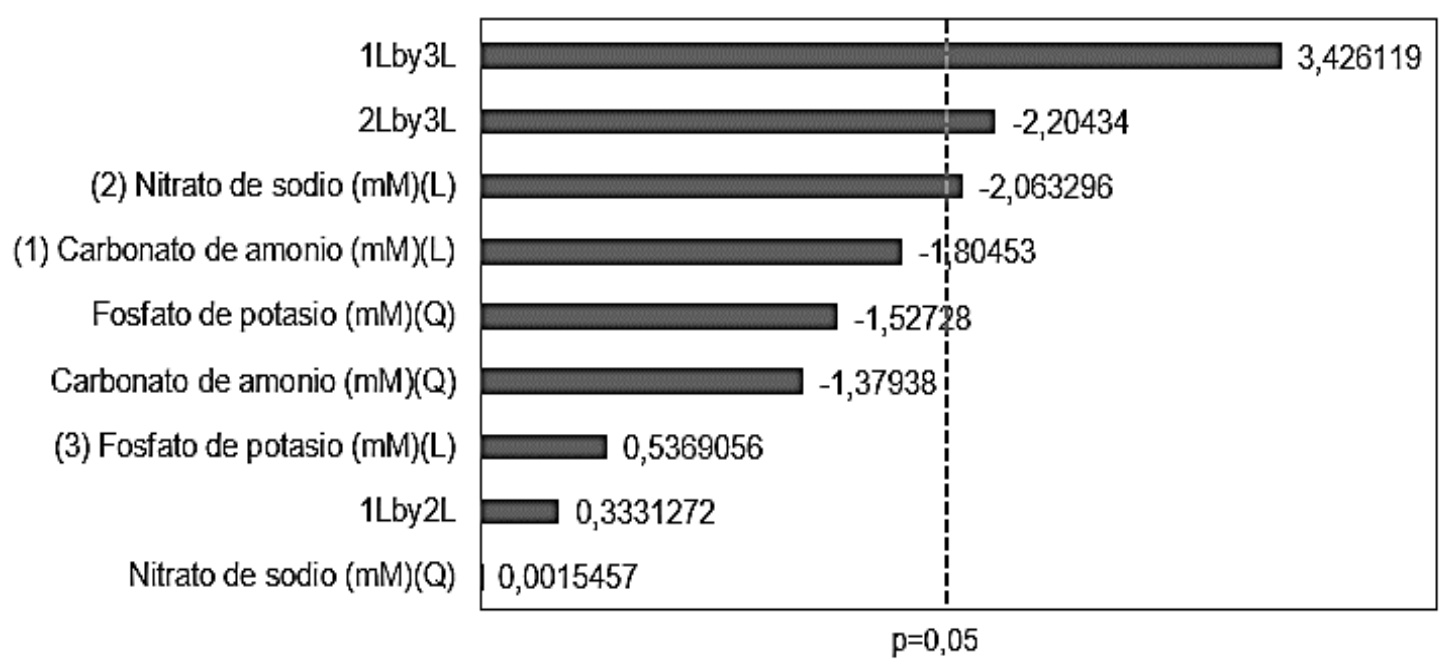

Efecto estandarizado estimado (Valor Absoluto)

(b)

Fig. 4. Diagrama de Pareto para la producción de lípidos con a) acetato de sodio y b) carbonato de potasio como fuente de carbono. Fuente: Autores.

Por otra parte, para el medio de cultivo con acetato, el mayor porcentaje de carbohidratos equivalente $73 \%$ de la biomasa se obtuvo el día 15 de cultivo con un tratamiento de $20 \mathrm{mM}$ acetato, $1,96 \mathrm{mM}$ nitrato y 1,15 $\mathrm{mM}$ fosfato, y para el cultivo con carbonato de amonio, se obtuvo $66 \%$ de carbohidratos a concentraciones de $1,074 \mathrm{mM}$ de carbonato de amonio, 0,98 $\mathrm{mM}$ de nitrato y 1,721 $\mathrm{mM}$ de fosfato. Finalmente, se alcanzó una concentración de lípidos del 0,34\% con 20
$\mathrm{mM}$ acetato, 0,32 $\mathrm{mM}$ nitrato y $1,15 \mathrm{mM}$ fosfato, mientras que $3,22 \mathrm{mM}$ de carbonato, $2,94 \mathrm{mM}$ de nitrato y $1,71 \mathrm{mM}$ de fosfato permitieron obtener $0,3 \%$ de lípidos, dado el bajo contenido de lípidos en comparación con el de carbohidratos, el aprovechamiento de esta biomasa debe estar orientada a las vías térmicas y de transformación de carbohidratos, y la vía lípidos se debe reservar a la presencia de ácidos grasos valiosos. 


\section{AGRADECIMIENTOS}

Los autores agradecen a la Universidad de Santander UDES, Universidad de Cartagena, Universidad Nacional Abierta y a Distancia y a la Universidad Industrial de Santander por proveer los equipos y software necesarios para realizar la presente investigación.

\section{REFERENCIAS}

[1] Á.-D. G.-D. and V. Kafarov, "Microalgae based biorefineries: issues to consider," Ciencia, Tecnol. y Futur., vol. 4, no. 4, pp. 522, 2011.

[2] T. M. Mata, A. A. Martins, O. Oliveira, S. Oliveira, A. M. Mendes, and N. S. Caetano, "Lipid content and productivity of arthrospira platensis and chlorella vulgaris under mixotrophic conditions and salinity stress," Chem. Eng. Trans., vol. 49, pp. 187$192,2016$.

[3] M.-K. Ji, H.-S. Yun, B. S. Hwang, A. N. Kabra, B.-H. Jeon, and J. Choi, "Mixotrophic cultivation of Nephroselmis sp. using industrial wastewater for enhanced microalgal biomass production," Ecol. Eng., vol. 95, pp. 527-533, Oct. 2016.

[4] L. Estévez, A. Barajas, C. Barajas, and V. Kafarov, "Improvement Of Lipid Productivity On Chlorella Vulgaris Using Waste Glycerol And Sodium Acetate," CT\&F - Ciencia, Tecnol. y Futur., vol. 5, no. 2, pp. 113-126, 2013.

[5] R. A. E. F. Hamouda, N. M. Sorour, and D. S. Yeheia, "Biodegradation of crude oil by Anabaena oryzae, Chlorella kessleri and its consortium under mixotrophic conditions," Int. Biodeterior. Biodegradation, vol. 112, pp. 128-134, Aug. 2016.

[6] J. Studer, J. Laue, and M. Koller, Bodendynamik: Grundlagen, Kennziffern, Probleme und Lösungsansätze, 3rd ed. Berlin: Springer, 2007.

[7] V. Bhola, R. Desikan, S. K. Santosh, K. Subburamu, E. Sanniyasi, and F. Bux, "Effects of parameters affecting biomass yield and thermal behaviour of Chlorella vulgaris," J. Biosci. Bioeng., vol. 111, no. 3, pp. 377-382, Mar. 2011.

[8] S. Zhu, W. Huang, J. Xu, Z. Wang, J. Xu, and Z. Yuan, "Metabolic changes of starch and lipid triggered by nitrogen starvation in the microalga Chlorella zofingiensis," Bioresour. Technol., vol. 152, pp. 292-298, Jan. 2014.
[9] K. Paranjape, G. B. Leite, and P. C. Hallenbeck, "Effect of nitrogen regime on microalgal lipid production during mixotrophic growth with glycerol," Bioresour. Technol., vol. 214, pp. 778-786, Aug. 2016.

[10] Y. Gao, M. Yang, and C. Wang, "Nutrient deprivation enhances lipid content in marine microalgae," Bioresour. Technol., vol. 147, pp. 484-491, Nov. 2013.

[11] K. Gautam, A. Pareek, and D. K. Sharma, "Biochemical composition of green alga Chlorella minutissima in mixotrophic cultures under the effect of different carbon sources," J. Biosci. Bioeng., vol. 116, no. 5, pp. 624-627, Nov. 2013.

[12] T.-S. Lin and J.-Y. Wu, "Effect of carbon sources on growth and lipid accumulation of newly isolated microalgae cultured under mixotrophic condition," Bioresour. Technol., vol. 184, pp. 100-107, May 2015.

[13] P. J. H. and M. M. W. R. A. Andersen, J. A. Berges, "Appendix A- Recipes for Freshwater and Seawater Media.," in In R.A. Andersen, A. C. Techniques, Ed. Burlington, MA, 2005, pp. 429-538.

[14] M. DuBois, K. A. Gilles, J. K. Hamilton, P. A. Rebers, and F. Smith, "Colorimetric Method for Determination of Sugars and Related Substances," Anal. Chem., vol. 28, no. 3, pp. 350-356, Mar. 1956.

[15] E. García-Martínez, B. Ayala-Torres, O. Reyes-Gómez, A. Zuorro, A. Barajas-Solano, and C. Barajas-Ferreira, "Evaluation of a two-phase extraction system of carbohydrates and proteins from chlorella vulgaris utex 1803," Chem. Eng. Trans., vol. 49, pp. 355-360, 2016.

[16] Y. Chen and S. Vaidyanathan, "A simple, reproducible and sensitive spectrophotometric method to estimate microalgal lipids," Anal. Chim. Acta, vol. 724, pp. 67-72, Apr. 2012.

[17] G. Dragone, B. D. Fernándes, A. P. Abreu, A. A. Vicente, and J. A. Teixeira, "Nutrient limitation as a strategy for increasing starch accumulation in microalgae," Appl. Energy, vol. 88, no. 10, pp. 3331-3335, Oct. 2011.

[18] Y. Liang, N. Sarkany, and Y. Cui, "Biomass and lipid productivities of Chlorella vulgaris under autotrophic, heterotrophic and mixotrophic growth conditions," Biotechnol. Lett., vol. 31, no. 7, pp. 1043-1049, Jul. 2009.

[19] B. Degrenne, J. Pruvost, G. Christophe, J. F. Cornet, G. Cogne, and J. Legrand, "Investigation of the combined effects of acetate and photobioreactor illuminated fraction in the induction of anoxia for hydrogen production by Chlamydomonas reinhardtii," Int. J. Hydrogen Energy, vol. 35, no. 19, pp. 10741-10749, Oct. 2010. 
[20] J. Fábregas, J. Abalde, and C. Herrero, "Biochemical composition and growth of the marine microalga Dunaliella tertiolecta (Butcher) with different ammonium nitrogen concentrations as chloride, sulphate, nitrate and carbonate," Aquaculture, vol. 83, no. 3-4, pp. 289-304, Dec. 1989.

[21] V. K. A. Y. Pinzón, Á. D. González-Delgado, "Optimization of microalgae composition for development of a topology of biorefinery based on profitability analysis," Chem. Eng. Trans., vol. 37, pp. 457-462, 20014.

[22] L. T. Porras Cárdenas and C. E. Prada
Rodríguez, "Efecto del tiempo de cultivo en la productividad de metabolitos de valor agregado en Chlorella vulgaris UTEX 1803," Universidad Industrial de Santander, 2012.

[23] Á. D. G.-D. V. Kafarov, M. El Halwagi, "Development of a topology of microalgaebased biorefinery: process synthesis and optimization using a combined forwardbackward screening and superstructure approach," Clean Technol. Environ. Policy, vol. 17, no. 8, pp. 2213-2228, 2015. 